\title{
Prime Factors and Divisibility of Sums of Powers of Fibonacci and Lucas Numbers
}

\author{
Spirit Karcher, Mariah Michael* \\ Department of Mathematics, Christopher Newport University, Newport News, VA \\ https://doi.org/10.33697/ajur.2020.036 \\ Students: spirit.karcher.13@cnu.edu,mariah.michael.16@cnu.edu* \\ Mentor: jessica.kelly@cnu.edu
}

\begin{abstract}
The Fibonacci sequence, whose first terms are $\{0,1,1,2,3,5, \ldots\}$, is generated using the recursive formula $F_{n+2}=$ $F_{n+1}+F_{n}$ with $F_{0}=0$ and $F_{1}=1$. This sequence is one of the most famous integer sequences because of its fascinating mathematical properties and connections with other fields such as biology, art, and music. Closely related to the Fibonacci sequence is the Lucas sequence. The Lucas sequence, whose first terms are $\{2,1,3,4,7,11, \ldots\}$, is generated using the recursive formula $L_{n+2}=L_{n+1}+L_{n}$ with $L_{0}=2$ and $L_{1}=1$. In this paper, patterns in the prime factors of sums of powers of Fibonacci and Lucas numbers are examined. For example, $F_{3 n+4}^{2}+F_{3 n+2}^{2}$ is even for all $n \in \mathbb{N}_{0}$. To prove these results, techniques from modular arithmetic and facts about the divisibility of Fibonacci and Lucas numbers are utilized.
\end{abstract}

\section{KEYWORDS}

Fibonacci Sequence; Lucas Sequence; Modular Arithmetic; Divisibility Sequence

\section{INTRODUCTION}

In the year 1202, Leonardo of Pisa, later known as Fibonacci, published a book entitled Liber Abacci or Book of Calculation which was one of the first European texts on algebra. ${ }^{1,2}$ Since Fibonacci was a merchant, the majority of his book focused on the Hindu-Arabic numerals and their practical applications in money conversions, profit margins, and other areas in commerce. However, at the end of Liber Abbaci, Fibonacci added a section of brain teasers and the solution to his most famous problem is still being studied today. The question posed was this:

If a pair of rabbits is placed in an enclosed area, how many pairs of rabbits will there be after a year if the following assumptions are made:

\section{Every month a pair of rabbits produces another pair}

2. Rabbits begin to bear young two months after their birth and

3. None of the rabbits die

To solve this problem, the pairs of rabbits at the end of each month are counted. Initially, one pair of rabbits is in the enclosed area $\left(F_{0}=1\right)$. After the first month, there will still be one pair of rabbits as the first pair is still unable to bear young $\left(F_{1}=1\right)$. After the second month, there will be two pairs of rabbits as the original pair will produce a pair of young $\left(F_{2}=2\right)$. After third month, there will be three pairs of rabbits as only one pair (the original) will be able to reproduce $\left(F_{3}=3\right)$. After the fourth month, there will be two pairs able to reproduce so there will be five total pairs $\left(F_{4}=5\right)$. After the fifth month, there will be three pairs able to reproduce so there will be eight total pairs $\left(F_{5}=8\right)$. Continuing in this manner, the pairs of rabbits may be counted for infinitely many months.

The sequence of numbers that results from this riddle is: 
This sequence was named the Fibonacci sequence after its creator and its terms became known as Fibonacci numbers. This sequence has numerous special properties and a variety of applications in the field of mathematics. Although it has been studied for centuries, the Fibonacci sequence continues to be a topic of study by mathematicians today. In fact, The Fibonacci Quarterly is a journal entirely dedicated to advances in mathematics related to Fibonacci numbers. ${ }^{3}$

Related to the Fibonacci sequence is the Lucas sequence. ${ }^{2}$ The Fibonacci and Lucas sequences share the same recursive formula but the initial conditions differ. These well-known sequences are two members of a larger family of recursive sequences. ${ }^{4-6}$ The focus of this paper is exploring patterns in the prime factors of sums of powers of Fibonacci and Lucas numbers.

\section{Background}

The recursive relationship of the Fibonacci sequence is formally defined as

$$
F_{n+2}=F_{n+1}+F_{n}, \text { for } n \in \mathbb{N}_{0} \text { with } F_{0}=0 \text { and } F_{1}=1 \text {. }
$$

Equation 2.

Notice that the the sequence in Equation 1. relates to the sequence defined in Equation 2 . by $F_{m}=F_{n+1}$ for $m \in \mathbb{N}_{0}$. The enumeration of the sequence defined in Equation 2. simplifies calculations and will be used for the remainder of the paper.

For the Lucas sequence, the recursive relationship is defined as

$$
L_{n+2}=L_{n+1}+L_{n}, \text { for } n \in \mathbb{N}_{0} \text { with } L_{0}=2 \text { and } L_{1}=1 \text {. }
$$

Equation 3 .

The Fibonacci and Lucas number sequences are two members of a larger family of recursive sequences. This larger family contains sequences called the general Lucas sequences of the first and second kind. ${ }^{\mathbf{4}-\mathbf{6}}$

For integers $P$ and $Q$, the $n$th term of general Lucas sequence of the first kind, $U_{n}(P, Q)$, is defined by

$$
\begin{gathered}
U_{n+2}(P, Q)=P U_{n+1}(P, Q)-Q U_{n}(P, Q), \text { for } n \in \mathbb{N}_{0} \\
\text { with } U_{0}(P, Q)=0 \text { and } U_{1}(P, Q)=1,
\end{gathered}
$$

while the $n$th term of a general Lucas sequence of the second kind, $V_{n}(P, Q)$, is defined by

$$
\begin{gathered}
V_{n+2}(P, Q)=P V_{n+1}(P, Q)-Q V_{n}(P, Q), \text { for } n \in \mathbb{N}_{0} \\
\text { with } V_{0}(P, Q)=2 \text { and } V_{1}(P, Q)=1 .{ }^{4}
\end{gathered}
$$

For $P=1$ and $Q=-1$, the Lucas sequence of the first kind is the Fibonacci numbers, while the Lucas sequence of the second kind is the Lucas numbers.

\section{Motivation}

The motivation for working with sums of powers of Fibonacci and Lucas numbers came from the results of Arangala et al. ${ }^{7}$ The authors explore finding closed form equations for sums of even powers of Fibonacci and Lucas numbers with indices that differ by two. For example,

$$
F_{n}^{2}+F_{n-2}^{2}=3 F_{n-1}^{2}+2(-1)^{n-1} .
$$

The proofs of Arangala et al. provide a foundation for useful techniques involving the identities and equations from above to manipulate sums of powers of Fibonacci numbers. The theorems were extended to Fibonacci numbers that had indices which differed by $k \equiv 0 \bmod 4$ rather than differing by 2 . For example, when extended to have the indices differ by $k \equiv 0 \bmod 4$ the result above becomes:

$$
F_{n}^{2}+F_{n-k}^{2}=L_{k} F_{n-\frac{k}{2}}^{2}+2 F_{\frac{k}{2}}^{2}(-1)^{n-\frac{k}{2}} \text { where } L_{k} \text { is the } k^{t h} \text { Lucas number. }
$$


Expanding on the ideas of Arangala et al., the results in this paper come from answering the following questions:

- Are there patterns in the prime factors of the sum $F_{n}^{2}+F_{n-2}^{2}$ for all $n \geq 2$ ?

- If so, what are they? And can these patterns be written in a way that holds for all $n \in \mathbb{N}_{0}$ ?

- How can the result for Fibonacci numbers be extended to the Lucas numbers and more generally, Lucas sequences of the first and second kind?

Studying results related to powers and the divisibility of Fibonacci and Lucas numbers is of particular interest to numerous mathematicians. ${ }^{5,6,8-16}$

\section{PRELIMINARIES}

Recall that given an integer $m$ and an integer $n$ such that $0 \leq n \leq m$, there exists integers $k$ and $r$ with $0 \leq r<n$ such that $m=k n+r$. The integer, $r$, is called the remainder modulo $n$. Working with the remainder $r$ as opposed to the number $m$ itself is called modular arithmetic. Modular arithmetic is similar to standard arithmetic in many ways but it does have a few special properties. In modular arithmetic there is not strict equality. Rather there is congruence of numbers using their remainders.

Definition: Let $n$ be a positive integer and let $a$ and $b$ be any integers. Then $a$ is congruent to $b$ mod $n$ written, $a \equiv b \bmod n$, if $a$ and $b$ have the same remainder when divided by $n$.

Modular arithmetic is useful when working with large integers because it simplifies problems by replacing an integer, $m$, with its remainder, $r$, when divided by a fixed positive integer, $n$.

Definition: An integer, $a$, is divisible by $n$, if $a \equiv 0 \bmod n$. Equivalently, if there exists an integer, $k$, such that $a=n k$.

For example, $7 \equiv 1 \bmod 2$ as $7=2(3)+1$; that is the remainder of 7 when divided by 2 is 1 . As well as, $10 \equiv$ 0 mod 2 since $10=2(5)+0$; the remainder is 0 . In other words, 10 is divisible by 2 .

Recall that all odd numbers can be written as $2 k+1$ where $k$ is an integer and all even numbers can be written as $2 k$ for some integer $k$. Then it follows that for any integer $m$ :

$$
m \equiv \begin{cases}0 \bmod 2 & \text { if } m \text { is even } \\ 1 \bmod 2 & \text { if } m \text { is odd }\end{cases}
$$

Three propositions related to modular arithmetic are presented below. Proposition 1 utilizes the definition for even and odd integers. Additionally, Propositions 2 and 3 concern sums and products under modular arithmetic. For the interested reader, additional results related to modular arithmetic may be found in any introduction discrete mathematics or number theory book. ${ }^{17-19}$

\section{Proposition 1.}

$i$. The sum of two odd numbers is an even number.

ii. The sum of two even numbers is an even number.

iii. The sum of an even number and an odd number is an odd number.

$i v$. The product of two even numbers is an even number.

$v$. The product of two odd numbers is an odd number.

vi. The product of an even number and an odd number is an even number. 
The proof for iii and vi are shown below and the proofs for the other results are left to the reader.

Proof. For iii:

Without loss of generality, let $n$ and $m$ be an even and odd number respectively. Then,

$$
n=2 k \text { and } m=2 l+1
$$

for integers $k$ and $l$. Then,

$$
n+m=2 k+(2 l+1)=(2 k+2 l)+1=2(k+l)+1 .
$$

Since the integers are closed under addition, $k+l$ is an integer. Therefore $n+m$ is an odd number.

Proof. For vi:

Without loss of generality, let $n$ be an even number and $m$ be an odd number. Then,

$$
n \cdot m=2 k(2 l+1)=4 k l+2 k=2(2 k l+k) .
$$

Since the integers are closed under addition, $2 k l+k$ is an integer. Therefore, $n m$ is an even number.

Addition of numbers under modular arithmetic works as expected. Computations may be done with only the remainders.

\section{Proposition 2.}

$$
(a \bmod n)+(b \bmod n) \equiv(a+b) \bmod n .
$$

Proof. Suppose there exist two numbers, such that the first is congruent to $a \bmod n$ and the second is congruent to $b \bmod n$; then the first is may be written as $n k+a$ and the second as $n l+b$ for integers $k$ and $l$. Then the sum of these two numbers under mod $n$ arithmetic is given by

$$
(a \bmod n)+(b \bmod n) \equiv(n k+a)+(n l+b) .
$$

The right hand side may be rewritten as

$$
\begin{aligned}
(n k+a)+(n l+b) & =(n k+n l)+(a+b) \\
& =n(k+l)+(a+b) .
\end{aligned}
$$

Therefore,

$$
(n k+a)+(n l+b) \equiv(a+b) \bmod n,
$$

and

$$
(a \bmod n)+(b \bmod n) \equiv(a+b) \bmod n
$$

from the definition of congruence $\bmod n$ from above.

Note that this property for addition under modular arithmetic provides an alternative proof for the properties (i)(iii) of even and odd numbers in Proposition 1. For example, the proof of Proposition 1(iii) is simplified to: For an odd number, $m$, and even number, $n, m \equiv 1 \bmod 2$ and $n \equiv 0 \bmod 2$, and so $m+n \equiv(1+0) \bmod 2 \equiv 1 \bmod 2$.

\section{Proposition 3.}

$(a \bmod n) \cdot(b \bmod n) \equiv(a \cdot b) \bmod n$. 
Note that this property for multiplication under modular arithmetic provides an alternative proof for the properties (iv)-(vi) of even and odd numbers in Proposition 1.

These propositions allow us to easily determine when a sum (or product) of Fibonacci and Lucas numbers is divisible by a given $n$. For example, using Equation 2., for any $F_{n}$ if the sum of the previous two is equivalent to $0 \bmod n$ then the sum is divisible by $n$.

From the properties of modular arithmetic, it may be observed that the Fibonacci numbers follow an interesting pattern under mod 2 arithmetic. As $F_{1}=F_{2}=1$, both are congruent to $1 \bmod 2$. From Proposition 1(i), the sum of two odd numbers is even; so $F_{1}+F_{2}=1+1 \equiv 0 \bmod 2$. However by Equation $2 ., F_{1}+F_{2}=F_{3}$. From here, $F_{2}+F_{3}$, is an odd number plus an even number so $F_{4} \equiv 1 \bmod 2$. The pattern of the Fibonacci numbers under mod 2 arithmetic continues throughout the entire sequence as follows:

$$
1,1,0,1,1,0,1,1,0,1,1,0, \ldots
$$

This observation that every third Fibonacci number is divisible by 2 demonstrates a much more powerful property of the Fibonacci numbers. It is a well known result that the Fibonacci numbers are an example of a divisibility sequence. ${ }^{10,11}$

Definition: A divisibility sequence is an integer sequence, $\left\{a_{n}\right\}$, indexed by positive integers $n$, such that if $m$ divides 1 then $a_{m}$ divides $a_{n}$.

There are many well known properties that follow from the Fibonacci numbers forming a divisibility sequence. These will be used to prove conjectures about the prime factors in sums of squares and cubes of Fibonacci numbers later in the paper.

\section{Proposition 4. $\quad$ i. Two divides every third Fibonacci number.}

ii. Three divides every fourth Fibonacci number.

iii. Five divides every fifth Fibonacci number.

Proof. To show (i), recall that $F_{3}=2$. From the definition of a divisibility sequence, this means that $F_{3}$ divides $F_{3 k}$ for all $k \in \mathbb{N}_{0}$. Therefore, all Fibonacci numbers whose index is divisible by 3 are divisible by 2 . To show (ii), recall that $F_{4}=3$. From the definition of a divisibility sequence, this means that $F_{4}$ divides $F_{4 k}$ for all $k \in \mathbb{N}_{0}$. Therefore, all Fibonacci numbers whose index is divisible by 4 are divisible by 3 . Lastly, to show (iii), recall that $F_{5}=5$. From the definition of a divisibility sequence, this means that $F_{5}$ divides $F_{5 k}$ for all $k \in \mathbb{N}_{0}$. Therefore, all Fibonacci numbers whose index is divisible by 5 are divisible by 5 .

The reader may observe interesting patterns in Tables 1 and 2 when looking at the Fibonacci numbers under addition and multiplication using modular arithmetic. The properties of addition allow us to move horizontally across the row of $F_{n} \bmod n$ in Tables 1 and 2; the patterns will continue indefinitely by Proposition 2. The properties of multiplication under modular arithmetic give us the column entries. For example, $F_{7}=13 \equiv 1 \bmod 2$. In order to find $F_{7}^{3}$ mod 2 , it is only necessary to look at $1^{3} \bmod 2$ which is much simpler than calculating $13^{3}$ and then reducing that value mod 2 .

While the Fibonacci numbers form a divisibility sequence, it is straightforward to show that this property is not applicable to the Lucas numbers or more general Lucas sequences. For example, while 2 divides $4, L_{2}=3$ does not divide $L_{4}=7$. Notice that $L_{2}=3$ does divide $L_{6}=18$ and $L_{10}=123$. The Lucas sequence is, however, an odd divisibility sequence.

Definition: An odd divisibility sequence is an integer sequence, $\left\{a_{n}\right\}$, indexed by positive integers $n$, such that if $n / m$ is an odd integer, then $a_{m}$ divides $a_{n}$. 


\begin{tabular}{|c||c|c|c|c|c|c|c|c|c|c|c|c|c|c|c|c|}
\hline$F_{n}$ & 1 & 1 & 2 & 3 & 5 & 8 & 13 & 21 & 34 & 55 & 89 & 144 & 233 & 377 & 610 & 987 \\
\hline$F_{n} \bmod 3$ & 1 & 1 & 2 & 0 & 2 & 2 & 1 & 0 & 1 & 1 & 2 & 0 & 2 & 2 & 1 & 0 \\
\hline$F_{n}^{2} \bmod 3$ & 1 & 1 & 1 & 0 & 1 & 1 & 1 & 0 & 1 & 1 & 1 & 0 & 1 & 1 & 1 & 0 \\
\hline$F_{n}^{3} \bmod 3$ & 1 & 1 & 2 & 0 & 2 & 2 & 1 & 0 & 1 & 1 & 2 & 0 & 2 & 2 & 1 & 0 \\
\hline
\end{tabular}

Table 1. Powers of $F_{n}$ under $\bmod 3$, with $n \geq 1$

\begin{tabular}{|c||c|c|c|c|c|c|c|c|c|c|c|c|c|c|c|c|}
\hline$F_{n}$ & 1 & 1 & 2 & 3 & 5 & 8 & 13 & 21 & 34 & 55 & 89 & 144 & 233 & 377 & 610 & 987 \\
\hline$F_{n} \bmod 5$ & 1 & 1 & 2 & 3 & 0 & 3 & 3 & 1 & 4 & 0 & 4 & 4 & 3 & 2 & 0 & 2 \\
\hline$F_{n}^{2} \bmod 5$ & 1 & 1 & 4 & 4 & 0 & 4 & 4 & 1 & 1 & 0 & 1 & 1 & 4 & 4 & 0 & 4 \\
\hline$F_{n}^{3} \bmod 5$ & 1 & 1 & 3 & 2 & 0 & 2 & 2 & 1 & 4 & 0 & 4 & 4 & 2 & 3 & 0 & 3 \\
\hline
\end{tabular}

Table 2. Powers of $F_{n}$ under $\bmod 5$, with $n \geq 1$

Being an odd divisibility sequence is a much weaker property than being a divisibility sequence. As such, many of the results applicable to the Fibonacci numbers are not relevant to the Lucas numbers. For example, our results for Fibonacci numbers cannot be directly extended to the Lucas numbers.

Bala presents several new sequences based upon Lucas sequences of the first and second kind that do form divisibility sequences. ${ }^{4}$ As an example, for a Lucas sequence of the second kind with $n \in \mathbb{N}_{0}$ and $r \in \mathbb{Z}$, the sequences:

$$
\begin{aligned}
& Z_{r n}^{1}=V_{r n}-V_{3 r n} \\
& Z_{r n}^{2}=V_{r n}-3 V_{3 r n}+3 V_{5 r n}-V_{7 r n} \\
& Z_{r n}^{3}=V_{r n}-5 V_{3 r n}+10 V_{5 r n}-10 V_{7 r n}+5 V_{9 r n}-V_{11 r n}
\end{aligned}
$$

each form a divisibility sequence. ${ }^{4}$ These "sequences of sequences" will be the focus of results for Lucas numbers.

\section{RESULTS}

Fibonacci Numbers

The main results in this section come from examining patterns in the prime factors of the sum $F_{k n}^{2}+F_{k n-2}^{2}$. This quantity, a sum of squared Fibonacci numbers, is of interest similar quantities were studied elsewhere, such as in Arangala et. al. ${ }^{7}$, where a related sum $F_{k n+l}^{2}+F_{m n+r}^{2}$ is studied. This sum has specific indices which will allow the sum to be divisible by certain primes for all $n \geq 0$. The patterns are observed in Tables 1 and 2. Additionally, the sum $F_{n}^{3}+F_{n-2}^{3}$ is examined for specific primes that are not contained in the factors of the sum $F_{n}^{2}+F_{n-2}^{2}$.

Observing the prime factors for $F_{n}^{2}+F_{n-2}^{2}$ for $n$ from 2 to 50 , the first pattern was found for sums containing 2 as a prime factor.

Lemma 1. For all $n \geq 0$,

$$
F_{3 n+4}^{2}+F_{3 n+2}^{2} \text { is divisible by } 2 .
$$

Proof. Begin by using the recursive definition in Equation 2. for the Fibonacci numbers to rewrite $F_{3 n+4}$ in terms of $F_{3 n+3}$ and $F_{3 n+2}$ :

$$
F_{3 n+4}^{2}+F_{3 n+2}^{2}=\left(F_{3 n+3}+F_{3 n+2}\right)^{2}+F_{3 n+2}^{2}
$$

then expanding and combining like terms,

$$
=F_{3 n+3}^{2}+2 F_{3 n+3} F_{3 n+2}+2 F_{3 n+2}^{2} .
$$

It is clear that $2 F_{3 n+3} F_{3 n+2}$ and $2 F_{3 n+2}^{2}$ are divisible by 2 and thus even numbers. In order for the entire sum to be even, $F_{3 n+3}^{2}$ must also be even.

From Proposition 4(i) on the divisibility of Fibonacci numbers, $F_{n}$ is even when $n$ is divisible by 3 . Clearly, $F_{3 n+3}$ 
is even for all $n \geq 0$ since $3 n+3$ is divisible by 3 for all $n \geq 0$. Lastly, if $F_{3 n+3}$ is even then so too is $F_{3 n+3}^{2}$ by Proposition 1(iv) on even and odd numbers.

Therefore, $F_{3 n+4}^{2}+F_{3 n+2}^{2}$ is the sum of even terms and is itself even for all $n \geq 0$.

The second prime which appears in regular intervals as a factor of $F_{n}^{2}+F_{n-2}^{2}$ is 5 . Unlike the factor of 2 , not only does the factor of 5 show up in a consistent pattern but it always shows up in the factorization of two consecutive $n$ values.

Lemma 2. For all $n \geq 0$,

$$
\begin{array}{ll}
\text { a. } & F_{5 n+3}^{2}+F_{5 n+1}^{2} \text { is divisible by } 5 . \\
\text { b. } & F_{5 n+4}^{2}+F_{5 n+2}^{2} \text { is divisible by } 5 .
\end{array}
$$

Proof. To prove the first statement, first recall from Table 2 that $F_{n}$ will be divisible by 5 if and only if $n \equiv 0$ mod 5 , or equivalently there is a $F_{5 n}$ term in the sum. So, begin by using the recursive definition of the Fibonacci numbers to rewrite $F_{5 n+3}$

$$
F_{5 n+3}^{2}+F_{5 n+1}^{2}=\left(F_{5 n+2}+F_{5 n+1}\right)^{2}+F_{5 n+1}^{2}
$$

By expanding and combining like terms, the righthand side becomes

$$
=F_{5 n+2}^{2}+2 F_{5 n+2} F_{5 n+1}+2 F_{5 n+1}^{2}
$$

Using Equation 2. again, rewrite $F_{5 n+2}^{2}$ as follows:

$$
=\left(F_{5 n+1}+F_{5 n}\right)^{2}+2 F_{5 n+2} F_{5 n+1}+2 F_{5 n+1}^{2}
$$

then again, expand and combine like terms:

$$
=3 F_{5 n+1}^{2}+F_{5 n}^{2}+2 F_{5 n+1} F_{5 n}+2 F_{5 n+2} F_{5 n+1}
$$

Lastly, rewrite the $F_{5 n+2}$ in the last term using Equation 2.. The goal of this process is to have either a coefficient of 5 or $F_{5 n}$ in each term. So, expanding and combining like terms:

$$
=5 F_{5 n+1}^{2}+F_{5 n}^{2}+4 F_{5 n+1} F_{5 n} .
$$

The first term is clearly a multiple of 5 . Since the second and third terms both have an $F_{5 n}$ this implies that they are divisible by 5 by Proposition $4\left(\right.$ iii). Therefore, $5 F_{5 n+1}^{2}+F_{5 n}^{2}+4 F_{5 n+1} F_{5 n} \equiv 0 \bmod 5$ as all three terms are divisible by 5 . The second result follows similarly.

Unlike the factor of 2, 5 appears in "pairs" for consecutive $n$. Using a numerical experiment, it is observed that 5 was a factor of the sum $F_{n}^{2}+F_{n-2}^{2}$ for $n=3,4,8,9,13,15, \ldots$ where the $n$ values coincide with the indices in Lemma 2. At first this pattern is seemingly random, however Table 2 can provide insight as to why this occurs. The pattern in the $F_{n}^{2}$ for $n \geq 1$ under $\bmod 5$ is as follows:

$$
1,1,4,4,0,4,4,1,1,0,1,1,4,4,0,4,4,1,1,0, \ldots
$$

So $F_{n}^{2}$ will be either 0,1 , or $4 \bmod 5$ for all $n \geq 1$, which means in order to have the sum be equivalent to $0 \bmod 5$ it will either have to occur when both terms are equivalent to $0 \bmod 5$ or when one term is equivalent to $1 \bmod 5$ and the other is equivalent to $4 \bmod 5$. Since the indices of the sum $F_{n}^{2}+F_{n-2}^{2}$ are two units apart, there will never have the case where both terms are equivalent to $0 \bmod 5$. However, based on the pattern above, it becomes clear that the terms which are equivalent to $1 \bmod 5$ and $4 \bmod 5$ are two units apart and appear in pairs resulting in pairs of sums which are divisible by 5 appearing at equal intervals. For example, when $n=3$ the sum is $F_{3}^{2}+F_{1}^{2} \equiv$ $1 \bmod 5+4 \bmod 5$ which from Proposition 2 is equivalent to $(1+4) \bmod 5 \equiv 0 \bmod 5$. 
So far a pattern in this sum with a prime factor of 2 as well as a very interesting "pairing" pattern for a prime factor of 5, has been observed. Curiously, based on initial observations 3 is not a factor of $F_{n}^{2}+F_{n-2}^{2}$ for $2 \leq n \leq 100$. Now it will be shown that 3 is not be a divisor of $F_{n}^{2}+F_{n-2}^{2}$ for all $n \in \mathbb{N}_{0}$.

Lemma 3. For all $n \geq 2$,

$$
F_{n}^{2}+F_{n-2}^{2} \text { will never be divisible by } 3 \text {. }
$$

Proof. This result will be proved by contradiction. Assume that $F_{n}^{2}+F_{n-2}^{2}$ has a factor of 3 , or equivalently, $F_{n}^{2}+$ $F_{n-2}^{2} \equiv 0 \bmod 3$. From the properties of addition under modular arithmetic this will happen when either:

a. $F_{n}^{2}$ and $F_{n-2}^{2}$ both have a factor of 3 ; that is, they are both congruent to $0 \bmod 3$, or

b. $F_{n}^{2}$ is congruent to $1 \bmod 3$ and $F_{n-2}^{2}$ is congruent to $2 \bmod 3$ or vice versa.

In case a: using Proposition 4(ii) and properties of modular arithmetic, $F_{n}$ will have a factor of 3 if and only if $n \equiv 0 \bmod 4$. Then, it follows that $F_{n}^{2}$ will also have a factor of 3 when $n \equiv 0 \bmod 4$. The same will hold for $F_{n-2}^{2}$. Without loss of generality, assume that $n-2 \equiv 0 \bmod 4$. Proposition 2 implies that $n \equiv 2 \bmod 4$. Therefore only one of $n$ or $n-2$ can be equivalent to $0 \bmod 4$. Thus, the assumption that $F_{n}^{2}+F_{n-2}^{2}$ has a factor of 3 leads to a contradiction, so $F_{n}^{2}+F_{n-2}^{2}$ does not have a factor of 3 .

So consider case b: assume that $F_{n}^{2} \equiv 1 \bmod 3$ and $F_{n-2}^{2} \equiv 2 \bmod 3$. Using Table 1 , observe that $F_{n}^{2} \not \equiv 2 \bmod 3$ for any $n$. This can be proved rigorously using Proposition 2 . Thus, the assumption that $F_{n}^{2} \equiv 1 \bmod 3$ and $F_{n-2}^{2} \equiv$ $2 \bmod 3$ (or vice versa) leads to a contradiction.

Therefore, the original assumption is false. Hence, $F_{n}^{2}+F_{n-2}^{2}$ will never have a factor of 3 for any integer $n \geq$ 2 .

Lemma 3 shows there will never be a factor of three in a sum of squares. However, it is conjectured that a factor of three may show up in a sum of cubes. Recall from Table 1 , that $F_{n}^{3}$ can be equivalent to 0 , 1, or, $2 \bmod 3$ so although, $F_{n}$ and $F_{n-2}$ cannot both be equivalent to $0 \bmod 3$, when considering a sum of cubes, there is now the possibility of having $F_{n}^{3} \bmod 1 \bmod 3$ and $F_{n-2}^{3} \bmod 2 \bmod 3$ or vice versa.

Lemma 4. For all $n \geq 0$,

$$
F_{4 n+3}^{3}+F_{4 n+1}^{3} \text { is divisible by } 3 .
$$

Proof. In order for $F_{4 n+3}^{3}+F_{4 n+1}^{3}$ to be divisible by 3, it is necessary to show that it can be written as a sum of terms which are all divisible by 3. Begin by using Equation 2. to rewrite the first term.

$$
F_{4 n+3}^{3}+F_{4 n+1}^{3}=\left(F_{4 n+2}+F_{4 n+1}\right)^{3}+F_{4 n+1}^{3}
$$

Expanding $\left(F_{4 n+2}+F_{4 n+1}\right)^{3}$ and then combining terms

$$
=F_{4 n+2}^{3}+3 F_{4 n+2}^{2} F_{4 n+1}+3 F_{4 n+2} F_{4 n+1}^{2}+2 F_{4 n+1}^{3}
$$

Again, using Equation 2. to rewrite $F_{4 n+2}^{3}$

$$
=\left(F_{4 n+1}+F_{4 n}\right)^{3}+3 F_{4 n+2}^{2} F_{4 n+1}+3 F_{4 n+2} F_{4 n+1}^{2}+2 F_{4 n+1}^{3}
$$

Expanding $\left(F_{4 n+1}+F_{4 n}\right)^{3}$ and combining terms

$$
=3 F_{4 n+1}^{3}+3 F_{4 n+1}^{2} F_{4 n}+3 F_{4 n+1} F_{4 n}^{2}+3 F_{4 n+2}^{2} F_{4 n+1}+3 F_{4 n+2} F_{4 n+1}^{2}+F_{4 n}^{3}
$$

It is clear that every term whose coefficient is 3 is divisible by 3 . Now check that $F_{4 n}^{3}$ is divisible by 3 for all $n$. Recall from Table 1 and divisibility that $F_{m}$ is divisible by 3 when $m$ is a multiple of 4 . Therefore, $F_{4 n}$ is divisi- 
ble by 3 for all $n$ and thus, so is $F_{4 n}^{3}$. Since $F_{4 n+3}^{3}+F_{4 n+1}^{3}$ is the sum of terms, each divisible by 3 , it too will be divisible by 3 .

\section{Lucas Numbers}

Extending the results of the last section to the Lucas numbers is not possible using the same techniques as the Lucas numbers do not form a divisibility sequence. The arguments of the work with Fibonacci numbers heavily rely on the fact that the Fibonacci numbers form a divisibility sequence.

To move around this difficulty, the results of Bala ${ }^{4}$ are utilized. Recall that the Lucas numbers are a Lucas sequence of the second kind with $P=1$ and $Q=-1$. This is denoted by $\left\{L_{n}\right\}_{n \in \mathbb{N}_{0}}=\left\{V_{n}(1,-1)\right\}_{n \in \mathbb{N}_{0}}$. In each of the theorems below, a divisibility sequence $\left\{Z_{r n}\right\}$ is created from terms of Lucas numbers. Then the focus is on the sequences, $\left\{W_{r n}\right\}$, made of sums of terms from $\left\{Z_{r n}\right\}$. In particular, the objective is to see when the terms will be divisible by two.

Theorem 1. Let $\left\{L_{n}\right\}$ be the Lucas sequence, and define $Z_{r n}=L_{r n}-L_{3 r n}$ and $W_{r n}=Z_{r n}^{2}+Z_{r(n-1)}^{2}$ for all $n \in \mathbb{N}_{0}$.

For all $n \geq 0$ and $r \equiv 0 \bmod 3, W_{r n}$ is divisible by 2 .

Proof. The aim is to show for all $n \in \mathbb{N}, W_{r n}$ will be even when $r=3 k$. In other words, $W_{r n} \equiv 0$ mod 2 if and only if $r \equiv 0 \bmod 3$. There are three cases to study: $r \equiv 0,1$ or $2 \bmod 3$.

Case $1(r \equiv 0 \bmod 3):$ When $r \equiv 0 \bmod 3, r n \equiv 0 \bmod 3$ and $r(n-1) \equiv 0 \bmod 3$. Therefore $L_{r n}, L_{3 r n}$, $L_{r(n-1)}$, and $L_{3 r(n-1)}$ will all be even for any $n \in \mathbb{N}_{0}$. Therefore, $Z_{r n}$ and $Z_{r(n-1)}$ will be even and $W_{r n}$ will also be even.

Case $2(r \equiv 1 \bmod 3):$ When $r \equiv 1 \bmod 3, r n \equiv 0 \bmod 3$ only when $n \equiv 0 \bmod 3$. Similarly, $r(n-1) \equiv 0$ $\bmod 3$ only when $n-1 \equiv 0 \bmod 3$. It is impossible to choose $n \in \mathbb{N}_{0}$ such that $n \equiv 0 \bmod 3$ and $n-1 \equiv 0$ mod 3. Therefore either $L_{r n}$ or $L_{r(n-1)}$ is odd. Hence either $Z_{r n}$ or $Z_{r(n-1)}$ is odd and consequently $W_{r n}$ is odd.

Case $3(r \equiv 2 \bmod 3):$ When $r \equiv 2 \bmod 3, r n \equiv 0 \bmod 3$ only when $n \equiv 0 \bmod 3$. Similarly, $r(n-1) \equiv 0$ $\bmod 3$ only when $n-1 \equiv 0 \bmod 3$. As in Case 2 , it is impossible to choose $n \in \mathbb{N}_{0}$ such that $n \equiv 0$ mod 3 and $n-1 \equiv 0 \bmod 3$. Therefore either $L_{r n}$ or $L_{r(n-1)}$ is odd. Hence either $Z_{r n}$ or $Z_{r(n-1)}$ is odd and consequently $W_{r n}$ is odd.

\section{DISCUSSION AND CONCLUSIONS}

The Fibonacci numbers have other well-known properties about divisibility that were not discussed in this paper. For example, 29 divides every $14^{\text {th }}$ Fibonacci number. This follows since $F_{14}=29$ and the Fibonacci sequence is a divisibility sequence. Using numerical results, it has been observed that $F_{n}^{2}+F_{n-2}^{2}$ when $n=5,11,19,25, \ldots$ so the authors conjecture that

$$
F_{14 n+5}^{2}+F_{14 n+3}^{2} \text { is divisible by } 29
$$

and

$$
F_{14 n+11}^{2}+F_{14 n+9}^{2} \text { is also divisible by } 29 .
$$

Similar to the "pairing" nature of sums with a factor of 5 , the values of $n$ for which $F_{n}^{2}+F_{n-2}^{2}$ has a factor of 29 are spaced out at repeating intervals of 6 then 8 which results in the two separate conjectures. It is also interesting to note that each pair of these intervals adds up to a difference in $n$ of 14 and this coincides with the divisibility property that states 29 will divide every $14^{\text {th }}$ Fibonacci number.

The results of this paper pertaining to the Lucas sequence could be further expanded by looking at other Lucas sequence-based divisibility sequences such as those found in the work of Bala ${ }^{\mathbf{4}}$ or Somer. ${ }^{\mathbf{5}, 6}$ For example generalizing the results for all integers $P$ and $Q$ and using the Lucas sequence of the second kind. Additionally, based on preliminary numerical experiments the following conjectures based are proposed: 
For all $n \geq 0$,

$$
L_{3 n+5}^{2}+L_{3 n+3}^{2} \text { is divisible by } 2 \text {. }
$$

and

$$
L_{n+3}^{3}+L_{n+1}^{3} \text { is divisible by } 5 \text {. }
$$

\section{ACKNOWLEDGMENTS}

The authors are grateful to the anonymous reviewers for their suggestions on improving the presentation and content of this paper.

\section{REFERENCES}

1. Katz, V. (1998) A history of mathematics: an introduction 2nd ed., Addison-Wesley.

2. Tannenbaum, P. (2014) Excursions in modern mathematics 8th ed., Pearson.

3. Fibonacci Quarterly, https://www.fq.math.ca/index.html, (accessed Dec 2020)

4. Bala, P. Lucas sequences and divisibility sequences, https://oeis.org/A273622/a273622.pdf, (accessed Feb 2016)

5. Somer, L. (1992) Divisibility of terms in Lucas sequences by their subscripts, in Applications of Fibonacci numbers (Bergum, G., Philippou, A., and Horadam A., Eds.) 515-525, Springer, Dordrecht. https://doi.org/10.1007/978-94-011-2058-6_52

6. Somer, L. (1996) Divisibility of terms in Lucas sequences of the second kind by their subscripts, in Applications of Fibonacci numbers (Bergum, G., Philippou, A., and Horadam A., Eds.) 473-486, Springer, Dordrecht. https://doi.org/10.1007/978-94-009-0223-7_39

7. Arangala, C., Hrovat, B., and Kelner, J. (2016) Sums of powers of Fibonacci and Lucas numbers. Minnesota J. of Undergrad. Math. 2 (1), 1-12.

8. Brillhart, J., Montgomery, P., and Silverman, R. (1988) Tables of Fibonacci and Lucas factorizations, Math. Comput., 50 (181), 251-260. https://doi.org/10.1090/S0025-5718-1988-0917832-6

9. Clary, S., and Hemenway, P. (1993) On sums of cubes of Fibonacci numbers, in Applications of Fibonacci numbers (Bergum, G., Philippou, A., and Horadam A., Eds.) 123-136, Springer, Dordrecht. https://doi.org/10.1007/978-94-011-2058-6_12

10. Gandhi, K. (2011) Divisibility properties of Fibonacci numbers. South Asian J. Math., 1 (3), 140-144.

11. Hoggatt, V., and Long, C. (1974) Divisibility properties of generalized Fibonacci polynomials. Fibonacci Quart. 12, 11-3-120.

12. Jaidee, M., and Pongsriiam, P. (2019) Arithmetic Functions of Fibonacci and Lucas Numbers. Fibonacci Quart., 57 (3), 246-254.

13. Melham, R. (2000) Alternating sums of fourth powers of Fibonacci and Lucas numbers. Fibonacci Quart. 38 (3), 254-259.

14. Melham, R. (2008/2009) Some conjectures concerning sums of odd powers of Fibonacci and Lucas numbers. Fibonacci Quart. 46/47 (4), 312-315.

15. Pongsriiam, P. (2014) Exact divisibility by powers of the Fibonacci and Lucas numbers, J. of Integer Seq. 17, 1-12. https://doi.org/10.3934/math.2020433

16. Thongmoon, M. (2009) New identities for the even and odd Fibonacci and Lucas numbers. Int. J. Contemp. Math. Sci. 4 (14), 671-676.

17. Jones, G., and Jones, J. (1998) Elementary number theory, Springer, London. https://doi.org/10.1007/978-14471-0613-5

18. Richmond, B. and Richmond, T. (2004) A discrete transition to advanced mathematics, Amer. Math. Soc., Rhode Island.

19. Sundstrom. T. (2013) Mathematical reasoning: writing and proof, https://scholarworks.gvsu.edu/books/24/, (accessed Dec 2020)

\section{ABOUT STUDENT AUTHORS}

Spirit Karcher graduated from Christopher Newport University in 2018. She is a member of the Pi Mu Epsilon, a national mathematics honor society; Alpha Delta Pi; and Order of Omega, a leadership honor society. Currently, Spirit is studying to earn her Ph.D. in mathematics education at Florida State University. 
Mariah Michael graduated from Christopher Newport University in 2020 with a B.S. in mathematics. She is a member of the Pi Mu Epsilon and Sigma Alpha Omega. She is currently studying to earn her M.S. in mathematics at George Mason University.

\section{PRESS SUMMARY}

This paper focuses on two specific types of recursive sequences; the Fibonacci sequence and Lucas sequence. The paper defines and examines the properties of divisibility and the sums of power of these two sequences. The work focuses on the patterns that result from manipulating the sequences. The work done in this paper can be expanded upon further by looking at higher sums of power in recursive sequences. 